\title{
Short term synaptic depression model—Analytical solution and analysis is $^{\text {is }}$
}

\author{
Joanna Mazurkiewicz ${ }^{\mathrm{a}, *, 1}$, Jarosław Żygierewicz ${ }^{\text {a }}$, Mikołaj Korzyński ${ }^{\text {b }}$ \\ ${ }^{a}$ Institute of Experimental Physics, Department of Biomedical Physics, Warsaw University, Hoża 69, 00-681 Warsaw, Poland \\ ${ }^{\mathrm{b}}$ Max-Planck-Institut für Gravitationsphysik (Albert-Einstein-Institut), Am Mühllberg 1, 14476 Golm, Germany
}

\section{A R T I C L E I N F O}

\section{Article history:}

Received 8 November 2007

Received in revised form

15 May 2008

Accepted 15 May 2008

Available online 21 May 2008

Keywords:

PPD

TM-model

\begin{abstract}
A B S T R A C T
In this article we present analytical solutions of the single and pair pulse time evolution of a plastic neocortical synapse described by the TM-model. We show that this model is equivalent to the receptordesensitization model with three kinetic states. For the TM-model we derive the analytical form of a measure of paired pulse depression. We analyze the sensitivity of the synaptic depression phenomenon on model parameters and derive the relative importance of each of the parameters. The closed form of the measure of synaptic depression allows fitting the model to experimental data. The fitted parameters are used to make predictions about the asymptotic properties of the postsynaptic currents. We show that for synapses with the ratio of inactivation and recovery rates of the same order, the synaptic depression does not preclude the rate-coding of information: e.g. in the pyramid-pyramid connections of adult rat neocortex, rate-coding is possible for higher frequencies.
\end{abstract}

๑) 2008 Elsevier Ltd. All rights reserved.

\section{Introduction-A model of neocortical synapse}

Synaptic transmission across neocortical synapses is strongly activity dependent (Thomson, 1997). Many phenomena like depression, augmentation and facilitation have been observed (Hempel et al., 2000; Thomson and Deuchars, 1994; Thomson, 1997; Zucker and Regehr, 2002). The effects of synaptic plasticity on properties of local circuits are yet to be investigated and therefore computational models of such synaptic properties are worth examining.

Short-term synaptic depression (STSD) is quite often observed and not fully understood. There are many mechanisms that can contribute to it. The most widespread of which seems to be a presynaptic decrease in the release of neurotransmitter that likely reflects a depletion of a release-ready pool of vesicles (Nadel et al., 1989). Other causes of synaptic depression are the release of modulatory substances from the activated presynaptic terminals, postsynaptic cells and neighboring cells. Finally, desensitization of ligand-gated receptors can make the target neuron less sensitive to neurotransmitter (Destexhe et al., 1994a, b).

In this article we focus on synaptic depression caused by the depletion of synaptic vesicles. In case of a slow tonic discharge a

\footnotetext{
The work was supported by the grant of Committee for Scientific Research to the Institute of Experimental Physics, Warsaw University.

* Corresponding author: Tel.: +48 22 5532126; fax: +48226226154.

E-mail addresses: asia@fuw.edu.pl (J. Mazurkiewicz), jarekz@fuw.edu.pl (J. Żygierewicz), Mikolaj.Korzynski@aei.mpg.de (M. Korzyński).

${ }^{1}$ The corresponding author has changed her name to Joanna Jẹdrzejewska-Szmek.
}

group of synaptic terminals, supplied by the same presynaptic axon and lying on a single postsynaptic target, may have the same probability of releasing transmitter in response to each action potential (AP). A proportion of these release sites would release transmitter in response to each postsynaptic AP. This proportion is relatively stable over many trials and can be described by a simple binomial distribution. In case of higher frequency of the stimulus, release site refractoriness (Betz, 1970; Stevens and Wang, 1995) limits the number of sites available for release to those that have been latent for some tens of milliseconds and those that have had time to recover from the last release (Thomson and Deuchars, 1994, 1997). Release probability is relatively high at many pyramidal connections and the pattern of release is dominated by release site refractoriness. In such connections one can observe STSD.

One of the first computational models reflecting synaptic depression was proposed in Tsodyks and Markram (1997) and is referred to as the TM-model. Its importance is mainly caused by two factors. The relative simplicity is the first factor. It only takes two differential equations to compute the postsynaptic current (PSC). The second factor is its versatility. It can account for two of the afore-mentioned mechanisms: depletion of synaptic vesicles and receptor desensitization. The TM-model characterizes the synaptic connection by its absolute amount of 'resources', which can be found in three states: effective-the resources taking part in the synaptic transfer, inactive-resources that are not accessible, and recovered-resources that are ready to take part in the synaptic transfer. Each presynaptic AP activates a certain fraction of the recovered resources-forming the effective fraction, which then quickly inactivates with a time constant of few milliseconds 


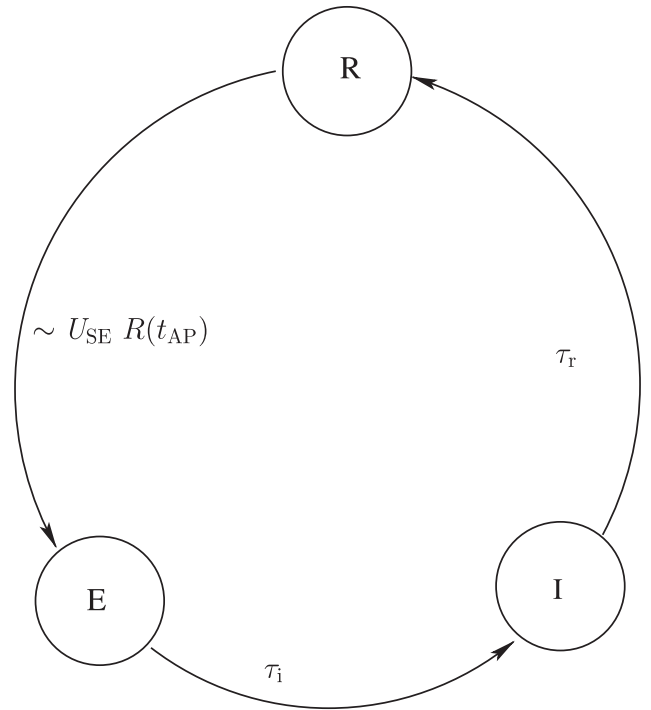

Fig. 1. Diagram of the model of short-term synaptic depression corresponding to Eq. (1). E (effective) - the resources taking part in the synaptic transfer, $I$ (inactive)-resources that are not accessible, and $R$ (recovered)-resources that are available. $\tau_{i}$ and $\tau_{r}$ are the time constants of inactivation and recovery, respectively. The utilization of synaptic efficacy, $U_{S E}$, describes the fraction of the resources in the recovered state, that is activated by an AP.

and then recovers with a time constant of at least several dozens of milliseconds ${ }^{2}$.

Visualization of the model is shown in Fig. 1 and kinetic equations are

$$
\begin{aligned}
\frac{\mathrm{d} R}{\mathrm{~d} t} & =\frac{I}{\tau_{r}}-U_{S E} R \delta\left(t-t_{A P}\right) \\
\frac{\mathrm{d} E}{\mathrm{~d} t} & =-\frac{E}{\tau_{i}}+U_{S E} R \delta\left(t-t_{A P}\right) \\
I & =1-R-E,
\end{aligned}
$$

where $E$ (effective), $I$ (inactive), $R$ (recovered) are the fractions of resources in corresponding states, while $\tau_{i}$ and $\tau_{r}$ are the time constants of inactivation and recovery, respectively. $U_{S E}$ (utilization of synaptic efficacy) describes the fraction of the resources in the recovered state, that is activated by an AP.

The net PSC, $I_{s y n}$, is proportional to the fraction of resources in the effective state, $E$ :

$I_{\text {syn }}=A_{S E} E$

where $A_{S E}$ is the absolute synaptic efficacy - the maximal possible response in case all the resources are activated by a presynaptic AP. The postsynaptic potential $V$ is given by

$\tau_{m} \frac{\mathrm{d} V}{\mathrm{~d} t}=-V+R_{\text {in }} I_{\text {syn }}$,

where $R_{\text {in }}$ denotes the input resistance of the postsynaptic membrane (resistance of the channels) and $\tau_{m}$ is the membrane's time constant. Substituting (3) into (2), one obtains

$\tau_{m} \frac{\mathrm{d} V}{\mathrm{~d} t}=-V+A_{S E} R_{\text {in }} E$

In order to find a form of the postsynaptic potential (PSP) one has to consider that $E$ in (4) is governed by (1) and solve the

\footnotetext{
${ }^{2}$ However, in real synapses the recovery from depression might be shorter than couple of dozens of milliseconds. In immature rats cerebellum recovery rates of about $13 \mathrm{~ms}$ have been found (Wall, 2005).
}

following set of differential equations:

$$
\begin{aligned}
& \frac{\mathrm{d} R}{\mathrm{~d} t}=\frac{1-R-E}{\tau_{r}}-U_{S E} R \delta\left(t-t_{A P}\right) \\
& \frac{\mathrm{d} E}{\mathrm{~d} t}=-\frac{E}{\tau_{i}}+U_{S E} R \delta\left(t-t_{A P}\right) \\
& \frac{\mathrm{d} V}{\mathrm{~d} t}=-\frac{V}{\tau_{m}}+\frac{A_{S E} R_{i n}}{\tau_{m}} E
\end{aligned}
$$

Analyzing (5) one expects that $R$ and $E$ are not continuous at $t_{A P}$. The product of the Dirac delta and a discontinuous function is indefinite. To make the model mathematically sensible the $\delta$ distribution has to be regularized. Depending on the regularization one can obtain different solutions. Thus (5) cannot be solved exactly.

\section{A finite version of the model}

Exact implementation of the Dirac delta function is impossible in silico. Usually one uses Kronecker delta (unit impulse) in order to model Dirac delta in simulations. It might, however, not suffice when one wants to shorten the simulation step and preserve the most important property of the Dirac delta:

$\int_{-\infty}^{\infty} \delta(x) \mathrm{d} x=1$

The simplest possible function which assures (6) is a step function whose amplitude is scaled with duration:

$s(t)= \begin{cases}\frac{1}{\Delta t}, & t \in(0, \Delta t) \\ 0 & \text { otherwise }\end{cases}$

The parameter $\Delta t$ can be seen as the duration of the AP or calcium, or generally as effective duration of the stimulation of the synapse. In this approach one substitutes the Dirac delta function with (7) obtaining

$$
\begin{aligned}
& \frac{\mathrm{d} R}{\mathrm{~d} t}=\frac{1-R-E}{\tau_{r}}-U_{S E} R s\left(t-t_{A P}\right) \\
& \frac{\mathrm{d} E}{\mathrm{~d} t}=-\frac{E}{\tau_{i}}+U_{S E} R s\left(t-t_{A P}\right) \\
& \frac{\mathrm{d} V}{\mathrm{~d} t}=-\frac{V}{\tau_{m}}+\frac{A_{S E} R_{i n}}{\tau_{m}} E
\end{aligned}
$$

One can also understand (8) as two sets of linear differential equations:

- During the time course of the $n$-th AP, $t \in\left(t_{A P_{n}}, t_{A P_{n}}+\Delta t\right)$ :

$$
\begin{aligned}
& \frac{\mathrm{d} R}{\mathrm{~d} t}=\frac{1-R-E}{\tau_{r}}-\frac{U_{S E}}{\Delta t} R \\
& \frac{\mathrm{d} E}{\mathrm{~d} t}=-\frac{E}{\tau_{i}}+\frac{U_{S E}}{\Delta t} R \\
& \frac{\mathrm{d} V}{\mathrm{~d} t}=-\frac{V}{\tau_{m}}+\frac{A_{S E} R_{i n}}{\tau_{m}} E
\end{aligned}
$$

- After $n$-th AP, $t \in\left(t_{A P_{n}}+\Delta t, t_{A P_{n+1}}\right)$ :

$$
\begin{aligned}
\frac{\mathrm{d} R}{\mathrm{~d} t} & =\frac{1-R-E}{\tau_{r}} \\
\frac{\mathrm{d} E}{\mathrm{~d} t} & =-\frac{E}{\tau_{i}} \\
\frac{\mathrm{d} V}{\mathrm{~d} t} & =-\frac{V}{\tau_{m}}+\frac{A_{S E} R_{i n}}{\tau_{m}} E
\end{aligned}
$$

In this form the model is equivalent to the model of receptor desensitization with three kinetic states introduced in Destexhe et al. (1994a, b). 
The synapse's response to two APs with a given inter-spike interval is an important characteristic of synaptic plasticity. Therefore we focused our attention on the problem of the synaptic reaction on two consecutive APs.

\section{Analytic solution of the model}

The sets of linear differential Equations (9) and (10) can be easily solved analytically. One obtains two sets of solutions-during the $n$-th AP and before the $(n+1)$-th AP with a boundary condition of continuity. After gluing the two solutions at the boundary, the solutions are of the form (11) and (13).

As the next step of analysis one can take the limit $\Delta t \rightarrow 0$ in all the functions of (11) in order to obtain the solutions of (5) with the regularization of the Delta function in the form (7). The kinetic functions $R$ and $E$ and the PSP during the $n$-th AP, $t \in\left(t_{A P_{n}}, t_{A P_{n}}+\Delta t\right)$ :

$$
\begin{aligned}
R(t) & =\frac{1}{\gamma}+A \mathrm{e}^{-(\beta+\alpha) \tau / 2 \tau_{r} \tau_{i}}+B \mathrm{e}^{-(\beta-\alpha) \tau / 2 \tau_{r} \tau_{i}} \\
E(t) & =\frac{\tau_{i} U_{S E}}{\gamma \Delta t}+C \mathrm{e}^{-(\beta-\alpha) \tau / 2 \tau_{r} \tau_{i}}+D \mathrm{e}^{-(\beta+\alpha) \tau / 2 \tau_{r} \tau_{i}} \\
V(t) & =V_{1} \mathrm{e}^{-\tau / \tau_{m}}+V_{c}+\mu \mathrm{e}^{-(\beta-\alpha) \tau / 2 \tau_{i} \tau_{r}}+v \mathrm{e}^{-(\beta+\alpha) \tau / 2 \tau_{i} \tau_{r}}
\end{aligned}
$$

where

$$
\begin{aligned}
& \tau=t-t_{A P_{n}} \\
& R_{<}=R\left(t_{A P_{n}}\right) \\
& E_{<}=E\left(t_{A P_{n}}\right) \\
& V_{<}=V\left(t_{A P_{n}}\right) \\
& R_{1}=R_{<}-\frac{1}{\gamma} \\
& E_{1}=E_{<}-\frac{\tau_{i}}{\gamma} \frac{U_{S E}}{\Delta t} \\
& V_{1}=V_{<}-V_{c}-\mu-v \\
& V_{c}=\frac{A_{S E} R_{m} \tau_{i} \frac{U_{S E}}{\Delta t}}{\gamma} \\
& A=\frac{R_{1}(\alpha+\eta)+2 \tau_{i} E_{1}}{2 \alpha} \\
& B=\frac{R_{1}(\alpha-\eta)-2 \tau_{i} E_{1}}{2 \alpha} \\
& C=\frac{2 \tau_{i} \tau_{r} \frac{U_{S E}}{\Delta t} R_{1}+E_{1}(\eta+\alpha)}{2 \alpha} \\
& D=\frac{\left(E_{1}(\alpha-\eta)-2 \tau_{i} \tau_{r} \frac{U_{S E}}{\Delta t} R_{1}\right)}{2 \alpha} \\
& \mu=\frac{A_{S E} R_{m}}{1-\frac{\beta-\alpha}{2 \tau_{r} \tau_{i}} \tau_{m}} C \\
& v=\frac{A_{S E} R_{m}}{1-\frac{\beta+\alpha}{2 \tau_{r} \tau_{i}} \tau_{m}} D \\
& \alpha=\sqrt{\tau_{i}^{2}+\tau_{r}^{2}\left(\frac{U_{S E}}{\Delta t} \tau_{i}-1\right)^{2}-2 \tau_{r} \tau_{i}\left(1+\frac{U_{S E}}{\Delta t} \tau_{i}\right)} \\
& \beta=\tau_{r}+\tau_{i}+\tau_{i} \tau_{r} \frac{U_{S E}}{\Delta t} \\
& \gamma=\left(1+\frac{U_{S E}}{\Delta t}\left(\tau_{r}+\tau_{i}\right)\right) \\
& \eta=\tau_{i}+\tau_{r}\left(\frac{U_{S E}}{\Delta t} \tau_{i}-1\right)
\end{aligned}
$$

The kinetic functions and the PSP after the offset of the $n$-th AP, $t \in\left(t_{A P_{n}}+\Delta t, t_{A P_{n+1}}\right):$

$$
\begin{aligned}
R(t) & =1+\left(R\left(t_{0}\right)-\frac{E\left(t_{0}\right) \tau_{i}}{\tau_{r}-\tau_{i}}-1\right) \mathrm{e}^{-\left(t-t_{0}\right) / \tau_{r}}+\frac{E\left(t_{0}\right) \tau_{i}}{\tau_{r}-\tau_{i}} \mathrm{e}^{-\left(t-t_{0}\right) / \tau_{i}} \\
E(t) & =E\left(t_{0}\right) \mathrm{e}^{-\left(t-t_{0}\right) / \tau_{i}} \\
V(t) & =\frac{E\left(t_{0}\right) \tau_{i} A_{S E} R_{m}}{\tau_{i}-\tau_{m}}\left(\mathrm{e}^{-\left(t-t_{0}\right) / \tau_{i}}-\mathrm{e}^{-\left(t-t_{0}\right) / \tau_{m}}\right)+V\left(t_{0}\right) \mathrm{e}^{-\left(t-t_{0}\right) / \tau_{m}} \\
t_{0} & =t_{A P_{n}}+\Delta t
\end{aligned}
$$

Obviously, in case of real neurons and synapses, most interesting phenomena are observed when repetitive APs arrive. The simplest case in which depression can be observed is when two APs arrive at the synapse with the inter-spike interval $T$. Some of the published results, e.g. Thomson (1997), are in the form of frequency dependent curves of the ratio of the two-spike PSP integral to the single-spike PSP integral. Let us denote the single-spike PSP integral as I-PSP-Integral and the two-spike PSP integral as II-PSP-Integral as in Thomson (1997).

It seems advisable to find the exact form of the PSP in the case of two APs with a given inter-spike interval and the two-spike PSP integral to single-spike PSP integral ratio. One can compare it with the experimental results avoiding the time-consuming simulations and determine the role and importance of different model parameters.

Let us assume that two APs arrive at the terminal of the synapse with an inter-spike interval of $T$. The synapse does not hold memory of the previous AP arrivals. Let us define the interspike interval as a period between the onset of the first AP and the onset of the second AP. The first AP arrives at the $t_{A P}$. There are four periods:

(1) $t \in\left(t_{A P}, t_{A P}+\Delta t\right)$ : PSP is described by $V(t)$ in (11). As the synapse does not hold memory of any previous spike arrivals the parameters are set to $V_{<}=0, E_{<}=0$ and $R_{<}=1$.

(2) $t \in\left(t_{A P}+\Delta t, t_{A P}+T\right)$ : PSP is described by $V(t)$ in (13). The solution has to be sewn with the previous solution (11) at $t_{A P}+\Delta t$. The sewing constants are $R_{1}=R\left(t_{A P}+\Delta t\right)$, $E_{1}=E\left(t_{A P}+\Delta t\right), V_{1}=V\left(t_{A P}+\Delta t\right)$.

(3) $t \in\left(t_{A P}+T, t_{A P}+T+\Delta t\right)$ : PSP is described by $V(t)$ in (11).The solution has to be sewn with the previous solution (13) at $t_{A P}+T$. The sewing constants $R_{2}, E_{2}, V_{2}$, should be calculated from (12).

(4) $t>t_{A P}+T+\Delta t$ : PSP is described by $V(t)$ in(13). The solution has to be sewn with the previous solution (11) at $t_{A P}+T+\Delta t$. The sewing constants are

$$
\begin{gathered}
R_{3}=R\left(t_{A P}+T+\Delta t\right), \quad E_{3}=E\left(t_{A P}+T+\Delta t\right), \\
V_{3}=V\left(t_{A P}+T+\Delta t\right) .
\end{gathered}
$$

\subsection{Measuring STSD}

A measure of pair pulse depression used e.g. by Thomson (1997) is the ratio of the integral of excitatory postsynaptic potential (EPSP) of two consecutive APs to the integral of EPSP of a single AP. Using analytical forms for $V(t)$, (11) and (13) the integrals can be also found in a closed form:

$$
\begin{aligned}
& \text { I-PSP-Integral } \\
& \begin{aligned}
= & \tilde{V} \tau_{m}\left(1-\mathrm{e}^{-\Delta t / \tau_{m}}\right)+V_{c} \Delta t+\mu \frac{2 \tau_{i} \tau_{r}}{\beta-\alpha}\left(1-\mathrm{e}^{-(\beta-\alpha) \Delta t / 2 \tau_{i} \tau_{r}}\right) \\
& +v \frac{2 \tau_{i} \tau_{r}}{\beta+\alpha}\left(1-\mathrm{e}^{-(\beta+\alpha) \Delta t / 2 \tau_{i} \tau_{r}}\right)+E_{1} A_{S E} R_{m}+V_{1} \tau_{m} \\
\tilde{V} & =-V_{c}-\mu-v
\end{aligned}
\end{aligned}
$$


and the initial conditions are $R_{<}=1, E_{<}=0, V_{<}=0$.

II-PSP-Integral

$$
\begin{aligned}
&= \tilde{V} \tau_{m}\left(1-\mathrm{e}^{-\Delta t / \tau_{m}}\right)+V_{c} \Delta t+\mu \frac{2 \tau_{i} \tau_{r}}{\beta-\alpha}\left(1-\mathrm{e}^{-(\beta-\alpha) \Delta t / 2 \tau_{i} \tau_{r}}\right) \\
&+v \frac{2 \tau_{i} \tau_{r}}{\beta+\alpha}\left(1-\mathrm{e}^{-(\beta+\alpha) \Delta t / 2 \tau_{i} \tau_{r}}\right) \\
&+\frac{E_{1} \tau_{i} A_{S E} R_{m}}{\tau_{i}-\tau_{m}}\left(\tau_{m}\left(\mathrm{e}^{-(T-\Delta t) / \tau_{m}}-1\right)+\tau_{i}\left(1-\mathrm{e}^{-(T-\Delta t) / \tau_{i}}\right)\right) \\
&+V_{1} \tau_{m}\left(1-\mathrm{e}^{-(T-\Delta t) / \tau_{m}}\right)+\tilde{V}_{2} \tau_{m}\left(1-\mathrm{e}^{-\left(\Delta t / \tau_{m}\right)}\right) \\
&+V_{c} \Delta t+\mu_{2} \frac{2 \tau_{i} \tau_{r}}{\beta-\alpha}\left(1-\mathrm{e}^{-(\beta-\alpha) \Delta t / 2 \tau_{i} \tau_{r}}\right) \\
&+v_{2} \frac{2 \tau_{i} \tau_{r}}{\beta+\alpha}\left(1-\mathrm{e}^{-(\beta+\alpha) \Delta t / 2 \tau_{i} \tau_{r}}\right)+E_{3} A_{S E} R_{m}+V_{3} \tau_{m} \\
& \tilde{V}_{=}= V_{c}-\mu-v \\
& \tilde{V}_{2}= V_{2}-V_{c}-\mu_{2}-v_{2} \\
& \mu=\mu \mu\left(R_{<}=1, E_{<}=0, V_{<}=0\right) \\
& v=v\left(R_{<}=1, E_{<}=0, V_{<}=0\right) \\
& \mu_{2}= \mu\left(R_{2}, E_{2}, V_{2}\right) \\
& v_{2}=v\left(R_{2}, E_{2}, V_{2}\right)
\end{aligned}
$$

Then the II-PSP-Integral to I-PSP-Integral depression ratio $(D R)$ is defined using (14) and (15) as

$D R=\frac{\text { II-PSP-Integral }}{\text { I-PSP-Integral }}$

$D R$ depends on the following set of parameters: Par $=\left\{A_{S E} R_{m}\right.$, $\left.\tau_{m}, \tau_{r}, \tau_{i}, U_{S E}, \Delta t, T\right\} . A_{S E} R_{m}$ is treated like a single parameter. The dependence of (16) on these parameters is complicated. Let us name the relative depression change depending on some parameter $p$ as $R D C_{p}$. It is introduced in (17) in order to estimate the influence of the parameters on $D R$ for each parameter. $R D C_{p}$ corresponds to the highest relative change of the depression ratio in parameter $p$ space, while the remaining parameters are fixed.

$R D C_{p}=\frac{\max _{p}(D R)-\min _{p}(D R)}{\overline{D R}}$

where $\overline{D R}$ denotes average $D R$ over $p$ space.

The global dependence of (16) on a given parameter can be characterized by maximizing $R D C_{p}$ over the other parameters.

$\max R D C_{p}=\max _{P a r / p}\left(R D C_{p}\right)$

The maximal $R D C_{p}$ was calculated for the following parameters $p$ : $A_{S E} R_{m}, \tau_{m}, \tau_{r}, \tau_{i}, U_{S E}, \Delta t$, and the inter-spike interval $T$. Estimated maximal $R D C_{p}$ (18) values obtained by scanning the parameter space Par and ranges of the parameters can be found in Table 1. The chosen parameter ranges are physiological and taken from Tsodyks and Markram (1997). The chosen $\Delta t$ range is broad to allow different time-scales.

Table 1

Values of the max $R D C_{p}$

\begin{tabular}{lll}
\hline Parameter & Range & Max RDC value \\
\hline$A_{S E} R_{m}$ & $0.1-100 \mathrm{mV}$ & 0 \\
$\tau_{m}$ & $1-100 \mathrm{~ms}$ & 0.10 \\
$\Delta t$ & $0.01-1 \mathrm{~ms}$ & 0.10 \\
$\tau_{i}$ & $1-13 \mathrm{~ms}$ & 0.15 \\
$\tau_{r}$ & $40 \mathrm{~ms}-1 \mathrm{~s}$ & 0.36 \\
$U_{S E}$ & $0.1-1.0$ & 0.50 \\
$T$ & $7 \mathrm{~ms}-1 \mathrm{~s}$ & 0.69 \\
\hline
\end{tabular}

It is clearly seen in Table 1 that the dependence of (16) on $\tau_{m}$ and $\Delta t$ is relatively small. $D R$ does not depend on $A_{S E} R_{m}$. The latter indicates that the model's dependence on the duration of synaptic stimulation is minimal and it might be advisable to investigate the case when $\Delta t \rightarrow 0$. In that case the number of parameters would decrease by one and most of the formulas derived in this article should simplify.

From Table 1 one can read that the $D R$ depends mostly on three parameters: $\tau_{r}, U_{S E}$ and the inter-spike interval. In order to show dependence of the $D R$ on chosen parameter $\left(\tau_{r}, U_{S E}\right.$ and $\left.\tau_{i}\right)$ and the inter-spike interval $T, D R(T)$ curves were drawn in Fig. 2. One can also see in Fig. 2(a) that the depression ratio decreases with increasing $\tau_{i}$.

One can see in Fig. 2(c) that the bigger the utilization of synaptic efficacy, the lower the depression ratio (16). The case of Fig. 2(b) is analogous, however, for smaller inter-spike intervals the depression ratio tends to have similar values independent of the value of $\tau_{r}$ as if the asymptotic values (as $T \rightarrow 0$ ) of the depression ratio did not depend on $\tau_{r}$. The role of $\tau_{r}$ diminishes for high frequency of the stimulus.

\section{Fitting the model to experimental data}

Some of the model parameters Par $=\left\{A_{S E} R_{m}, \tau_{m}, \tau_{r}, \tau_{i}, U_{S E}, \Delta t\right\}$ can be measured directly. The recovery time constant $\tau_{r}$ can be estimated from the time needed for the depression effect to vanish, the parameters $A_{S E} R_{m}$ and $\tau_{m}$ can be adjusted so that the EPSP amplitude and rise-time agree with those measured experimentally or calculated using the membrane conductance and resistance values. The remaining parameters $\tau_{i}$ and $U_{S E}$ are difficult to measure directly. One can, however, estimate them fitting the formula (16) to the experimental ratios of two-spike to one spike EPSP integral.

We present here the fit to the data shown in Fig. 2 of Thomson (1997). These experimental data were obtained in the paired pulse depression experiment performed on pyramid-pyramid connections of adult male rats. The pyramid-pyramid connections came from different layers and areas of the neocortex. The linearregression performed for these data indicates that this form of depression lasts for 60-70 ms (Thomson, 1997).

In the fit the duration of the stimulation of synapse $\Delta t$ was interpreted as the duration of an AP and set as $1 \mathrm{~ms}$. Following the data from Thomson (1997) we set $\tau_{r} \approx 65 \mathrm{~ms}$, the amplitude of EPSP to be $(1.4 \pm 1.1) \mathrm{mV}$ and the EPSP rise-time to be $(2 \pm 0.7) \mathrm{ms}$.

The remaining parameters $\tau_{i}$ and $U_{S E}$ were scanned in their physiological regions $\left(U_{S E} \in(0.15,0.95)\right.$, Tsodyks and Markram (1997), $\tau_{i}$ in the range of $1-30 \mathrm{~ms}$ ). For each scan of the $\tau_{i}$ and $U_{S E}$ parameter space, $A_{S E} R_{m}$ and $\tau_{m}$ were adjusted to fit the amplitude of EPSP and the EPSP rise-time. The best-fit parameters minimize the squared residuals. The uncertainty of determined parameters was calculated using the standard method, the second derivative of the sum of squared residuals.

The best-fit curve and experimental data can be found in Fig. 3. Parameters of the fit are presented in Table (2). We found $U_{S E} \approx$ 0.72 with $68 \%$-confidence in $(0.36,0.95)$, and $\tau_{i} \approx 12 \mathrm{~ms}$ with $68 \%$ confidence in $(1,37) \mathrm{ms}$. Both parameters $U_{S E}$ and $\tau_{i}$ exhibit such enormous uncertainty values due to the dispersion of the experimental data.

\section{Stationary amplitudes of PSC and the kinetic function $E$}

One of the most important predictions of Tsodyks and Markram (1997) is that the stationary PSC amplitude decays with 
a

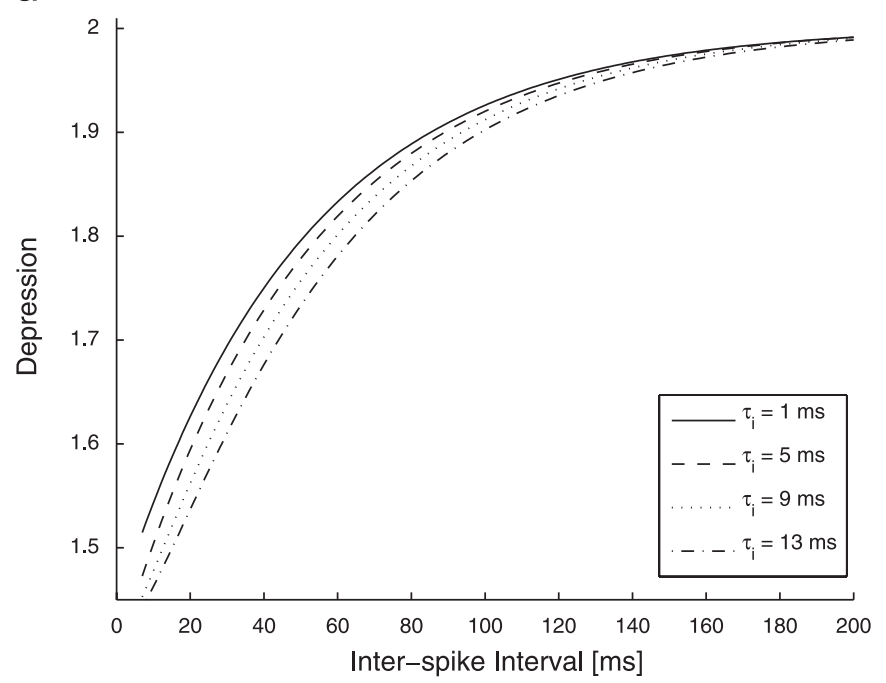

b

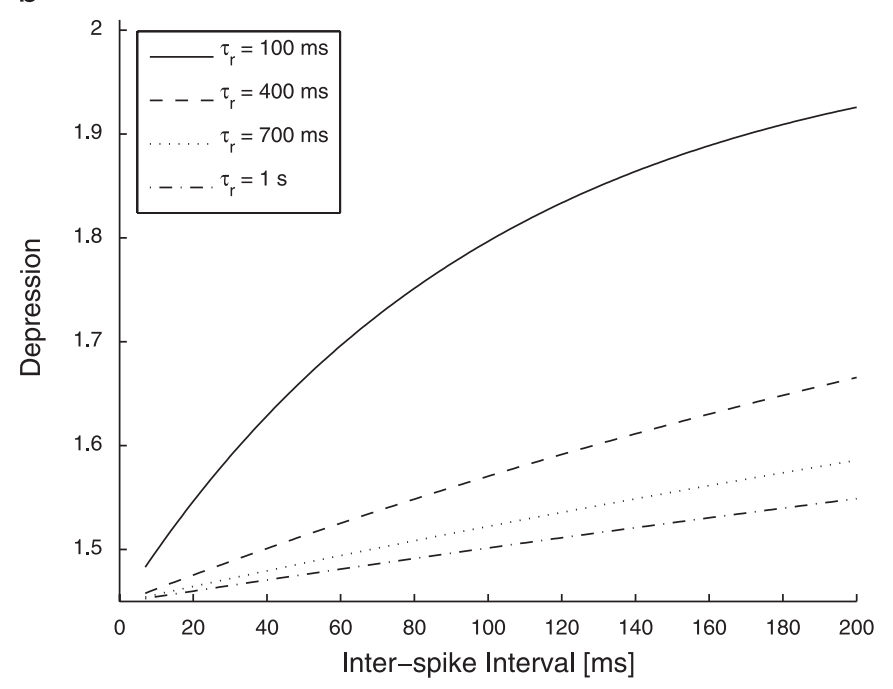

C

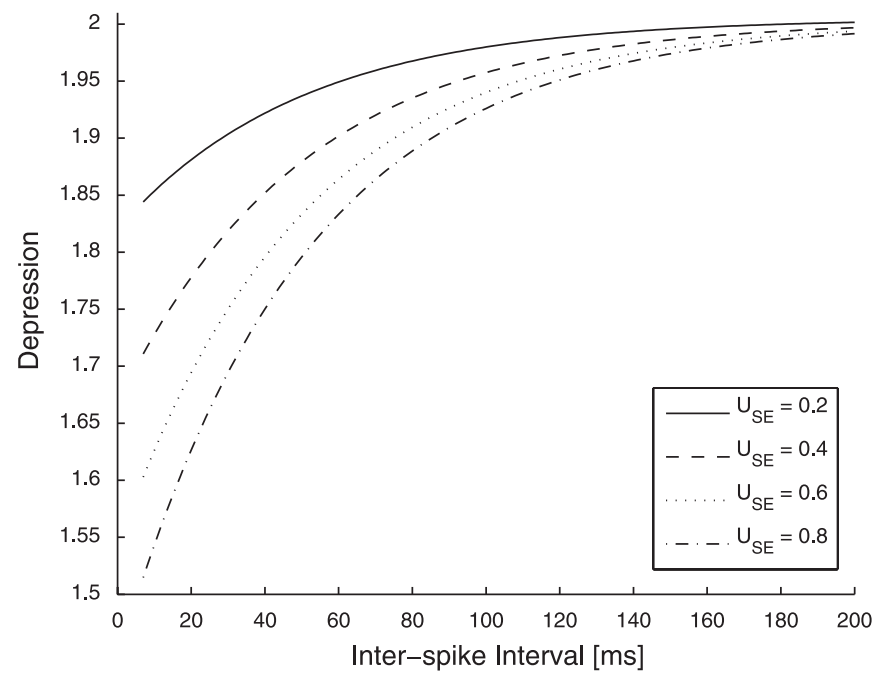

Fig. 2. Depression curves: dependence of depression ratio (16) on parameters of the model: (a) dependence on $\tau_{i}$, parameters: $\tau_{r}=50 \mathrm{~ms}, \tau_{i} \in\{1 \mathrm{~ms}, 5 \mathrm{~ms}, 9 \mathrm{~ms}$, $13 \mathrm{~ms}\}, \tau_{m}=20 \mathrm{~ms}, U_{S E}=0.8, A_{S E} R_{m}=1 \mathrm{mV}, \Delta t=1 \mathrm{~ms}$; (b) dependence on $\tau_{r}$, parameters: $\tau_{i}=1 \mathrm{~ms}, \tau_{r} \in\{100 \mathrm{~ms}, 400 \mathrm{~ms}, 700 \mathrm{~ms}, 1 \mathrm{~s}\}, \tau_{m}=20 \mathrm{~ms}, U_{S E}=0.8$ $A_{S E} R_{m}=1 \mathrm{mV}, \Delta t=1 \mathrm{~ms}$; (c) dependence on $U_{S E}$, parameters: $\tau_{r}=50 \mathrm{~ms}$, $\tau_{i}=1 \mathrm{~ms}, \tau_{m}=20 \mathrm{~ms}, U_{S E} \in\{0.2,0.4,0.6,0.8\}, A_{S E} R_{m}=1 \mathrm{mV}, \Delta t=1 \mathrm{~ms}$.

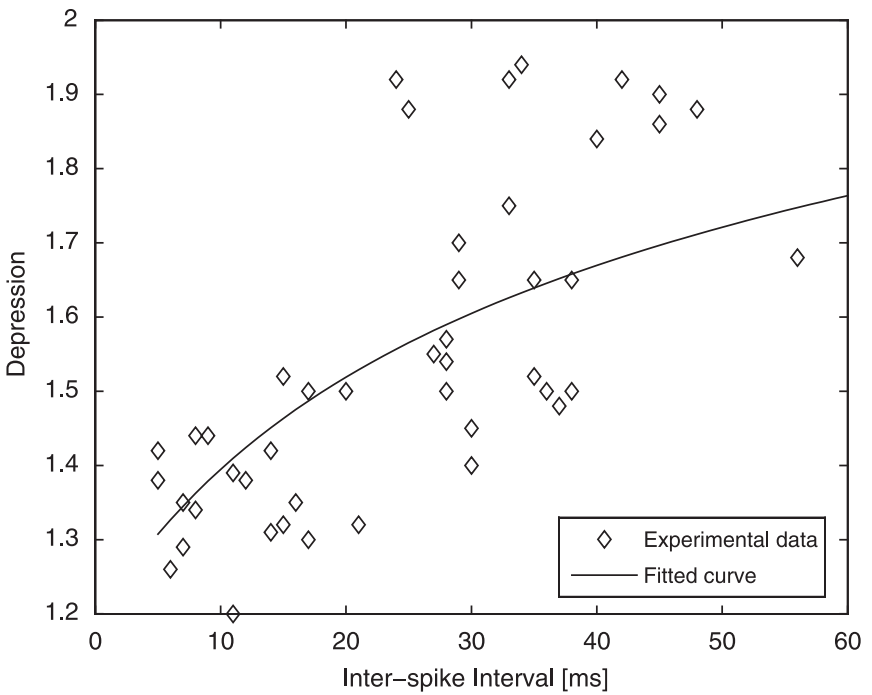

Fig. 3. The experimental data in Thomson (1997) and the best-fit curve. The fit was performed with fixed $\tau_{r}=65 \mathrm{~ms}$. Parameters of the fit are presented in Table 2 .

frequency of stimulation as $1 / f$. The PSC amplitude is related to the kinetic function $E$ by (2). Assuming $\tau_{i}$ much smaller than both $\tau_{r}$ and the inter-spike interval the stationary $E_{s t}$ was described as

$\forall>f_{\text {lim }} E_{s t}(f) \approx \frac{1}{\tau_{r} f}$

with the limiting frequency specified as

$f_{\text {lim }}=\frac{1}{\tau_{r} U_{S E}}$

Such property of the synapse precludes the frequency of stimulation as the information carrier.

In order to check if solutions of (11) and (13) also follow (19), we have derived the stationary amplitudes of the kinetic function $E$ in function of frequency. The formula describing the frequencydependent stationary $E_{s t}$ is of the form

$E_{s t}(f)=\frac{\tau_{i} U_{S E}}{\gamma \Delta t} \frac{\text { numerator }}{\text { denominator }}$

The numerator and denominator from Eq. (21) are presented below:

$$
\begin{aligned}
\text { numerator }= & \alpha^{2} \Delta t\left(x_{1}+x_{2}-2\right)\left(\left(x_{1}+x_{2}\right) x_{3}-2\right) \\
& -2 \alpha \Delta t\left(x_{1}-x_{2}\right)\left(x_{3}-1\right)\left(\eta-2(\gamma-1) \tau_{r}\right) \\
& +\left(x_{1}-x_{2}\right)^{2} x_{3}\left(4 \tau_{i}^{2} \tau_{r} U_{S E}-\Delta t \eta^{2}\right)
\end{aligned}
$$

denominator

$$
\begin{aligned}
= & 4 \tau_{i}^{2} \tau_{r} U_{S E}\left(x_{1}-x_{2}\right)\left(\frac{\alpha}{\tau_{i}-\tau_{r}}\left(x_{3}-x_{4}\right)+\left(x_{1}-x_{2}\right) x_{3} x_{4}\right) \\
& +\Delta t\left(\alpha\left(\left(x_{1}+x_{2}\right) x_{3}-2\right)+\left(x_{1}-x_{2}\right) x_{3} \eta\right) \\
& \times\left(\alpha\left(\left(x_{1}+x_{2}\right) x_{4}-2\right)-\left(x_{1}-x_{2}\right) x_{4} \eta\right)
\end{aligned}
$$

$x_{1}, x_{2}, x_{3}$ and $x_{4}$ are of the form:

$x_{1}=\mathrm{e}^{-(\beta+\alpha) \Delta t / 2 \tau_{r} \tau_{i}}$

$x_{2}=\mathrm{e}^{-(\beta-\alpha) \Delta t / 2 \tau_{r} \tau_{i}}$

$x_{3}=\mathrm{e}^{-1 / f \tau_{r}}$

$x_{4}=\mathrm{e}^{-1 / f \tau_{i}}$

and the constants $\alpha, \beta, \gamma, \eta$ are defined in Eq. (12).

Fig. 4 shows dependence of $E_{s t}(21)$ on $f$. For the parameters of synapses given in Tsodyks and Markram (1997) the $E_{s t}(f)$ plots 
Table 2

Model parameters, fit with fixed $\tau_{r}$

\begin{tabular}{|c|c|c|c|}
\hline Parameter & Value & $68 \%$ confidence in & Source \\
\hline$\Delta t$ & $1 \mathrm{~ms}$ & - & AP duration \\
\hline$\tau_{i}$ & $12 \mathrm{~ms}$ & $(1,32) \mathrm{ms}$ & Free parameter \\
\hline$\tau_{r}$ & $65 \mathrm{~ms}$ & - & (Thomson, 1997) \\
\hline$U_{S E}$ & 0.70 & $(0.36,0.95)$ & Free parameter (Tsodyks and Markram, 1997) \\
\hline$\tau_{m}$ & $1 \mathrm{~ms}$ & $(1,3) \mathrm{ms}$ & Fitted to (Thomson, 1997) \\
\hline$A_{S E} R_{m}$ & $3.5 \mathrm{mV}$ & $(3.4,3.6) \mathrm{mV}$ & Fitted to (Thomson, 1997) \\
\hline
\end{tabular}

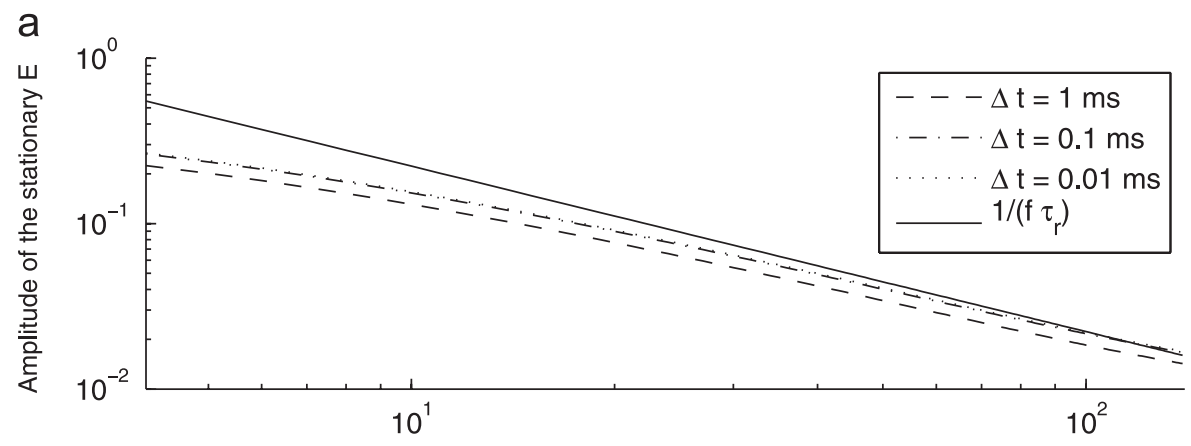

Frequency of the stimulus [Hz]

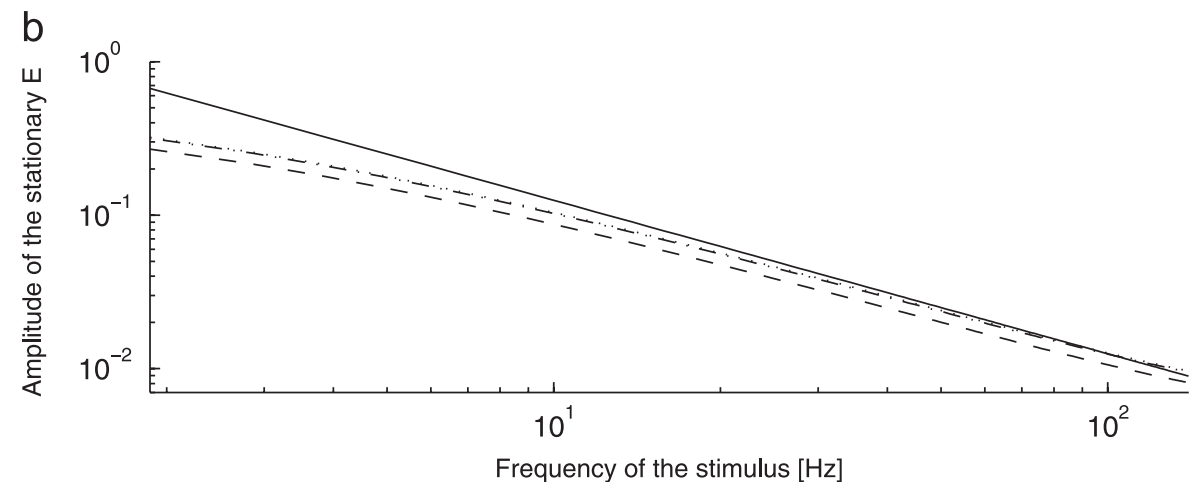

C

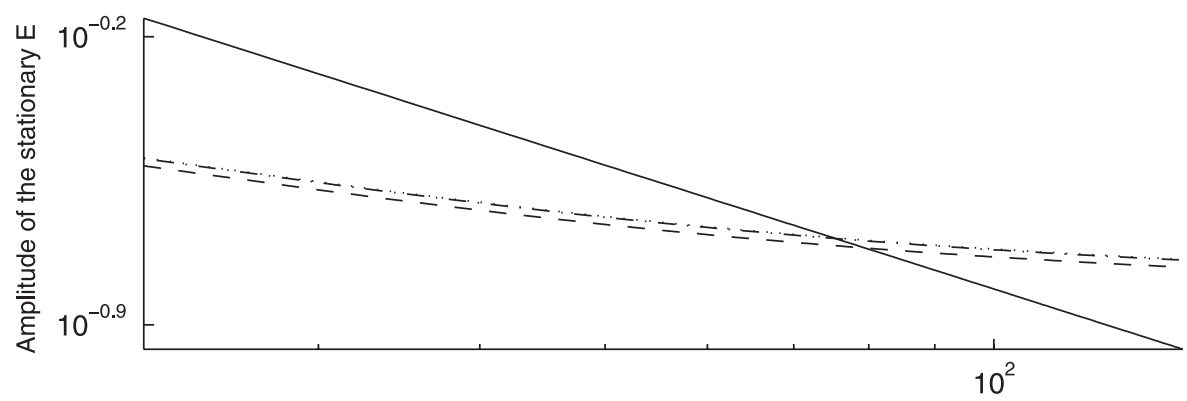

Frequency of the stimulus $[\mathrm{Hz}]$

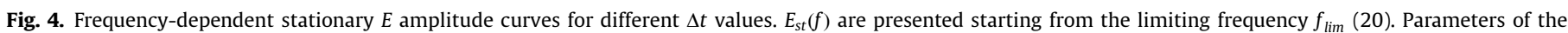

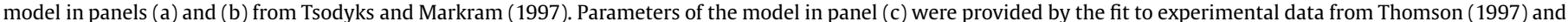

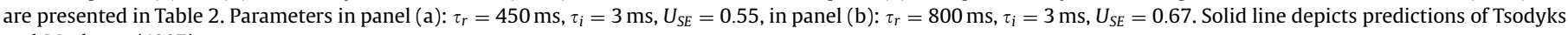
and Markram (1997).

show a pronounced decay with increasing frequency. These results agree with Tsodyks and Markram (1997).

However, in case of higher $\tau_{i}$ values $\left(\tau_{i}>3 \mathrm{~ms}\right) E_{s t}(f)$ curves show pronounced decay to an asymptotic non-zero value, $E_{A S}$. As one can see in Fig. $4(\mathrm{c})$ this asymptotic value can be half of $E_{\text {st }}\left(f_{\text {lim }}\right)$ and is reached for $f \approx 60 \mathrm{~Hz}$. As the parameter values of curves in panel (c) of Fig. 4 were obtained in a fit of experimental data, this might indicate that at the pyramid-pyramid connections infor- mation can be effectively rate-coded even for frequencies higher than $60 \mathrm{~Hz}$.

As the pulse-frequency $f$ goes to infinity, $E_{s t}(f)$ takes the limiting form of

$$
E_{A S}=\frac{\tau_{i}}{\frac{\Delta t}{U_{S E}}+\left(\tau_{r}+\tau_{i}\right)}
$$


When $\tau_{i} \ll \tau_{r}, E_{A S}$ is very small and in accordance with (19). However, in case of $\tau_{i}$ and $\tau_{r}$ of the same order of magnitude, $E_{A S}$ is considerable. This is illustrated in panel (c) of Fig. 4.

\section{Discussion}

We showed that step-regularization (7) of Dirac delta in the TM-model transforms the TM-model to receptor-desensitization model (Destexhe et al., 1994a, b). Thus with the activation of the form of (7) both models are formally equivalent. Hence, (16), (21) and (24) are applicable and can be used to make predictions for both models.

Using the exact expression (11) and (13) we derived a measure of synaptic depression based on the ratio of the integral of PSP induced by two APs to the integral of PSP induced by a single AP (16). This measure is compatible with the one used in literature in reporting the experimental results by e.g. Thomson (1997). The depression measure (16) depends on six model parameters. We proposed a method to characterize the role and quantitatively express the importance of each of the parameters in the synaptic depression. We showed that the most important parameters are $U_{S E}, T, \tau_{r}$ and $\tau_{i}$.

In the presented model there is no visible inter-spike interval threshold for the depression effect. The curves obtained from (16) are smooth. The explicit form of $D R(16)$ and the analysis of the importance of model parameters with $\max R D C_{p}$ allows for efficient fitting of the model to experimental data that are presented in the form of II-PSP-Integral to I-PSP-Integral curves.

Results of Sections 4 and 5 indicate that pyramid-pyramid cortical synapses exhibit depression for which the assumption that $\tau_{r}$ is at least a couple of dozen times bigger than $\tau_{i}$, does not hold. Also hippocampal data (Stevens and Wang, 1995) indicate that $\tau_{r}$ might be even smaller than $50 \mathrm{~ms}$, due to the fact that after the release site has been used a $20 \mathrm{~ms}$ interval is required to refill the site before it can be used again.

The model can also be seen as the desensitization of ligandgated receptors. In that case the recovery from desensitization can be around $13 \mathrm{~ms}$ (immature rats, Wall, 2005). Desensitization rate constants of different types of AMPARs subunits also point out that $\tau_{i}$ might be higher than $5 \mathrm{~ms}$ and range up to a dozen of milliseconds (Wall, 2005; Grosskreutz et al., 2003; Quirk et al., 2004). This suggests that $\tau_{i}$ can be of the same order as $\tau_{r}$. In such a case, the asymptotic values of the stationary current amplitudes (24) are non-zero and can be of a considerable magnitude. This does allow the depressible synapses of similar recovery and inactivation rates to transfer rate-coded information also at high frequencies.

\section{Acknowledgment}

The authors would like to thank Piotr Suffczyński, Jonathan Roberts and Zbigniew Jȩdrzejewski-Szmek for their constructive remarks.

\section{References}

Betz, W.J., 1970. Depression of transmitter release at the neuromuscular junction. J. Physiol. 206, 795.

Destexhe, A., Mainen, Z.F., Sejnowski, T.J., 1994a. Synthesis of models for excitable membranes, synaptic transmission and neuromodulation using a common kinetic formalism. J. Comput. Neurosci. 1 (3), 195.

Destexhe, A., Mainen, Z.F., Sejnowski, T.J., 1994b. Synthesis of models for excitable membranes, synaptic transmission and neuromodulation using a common kinetic formalism. Neural Comput. 6, 14.

Grosskreutz, J., Zoerner, A., Schlesinger, F., Krampfl, K., Dengler, R., Bufler, J., 2003. Kinetic properties of human ampa-type glutamate receptors expressed in hek293 cells. Eur. J. Neurosci. 27, 1173.

Hempel, C.M., Hartman, K.H., Wang, X.-J., Turrigano, G.G., Nelson, S.B., 2000. Multiple forms of short-term plasticity at excitatory synapses in rat medial prefrontal cortex. J. Neurophysiol. 83 (5), 3031.

Nadel, L., Cooper, L., Culicover, P., Harnish, R.M. (Eds.), 1989. Neural Connections, Mental Computation. MIT Press, Cambridge, MA.

Quirk, J.C., Siuda, E.R., Nisenbaum, E.S., 2004. Molecular determinants responsible for differences in desensitization kinetics of ampa receptor splice variants. European J. Neurosci. 24 (50), 11416.

Stevens, C.F., Wang, Y., 1995. Facilitation and depression at single central synapse. Neuron 14, 795.

Thomson, A.M., 1997. Activity-dependent properties of synaptic transmission at two classes of connections made by rat neocortical pyramidal axons in vitro. J. Physiol. 502 (1), 137.

Thomson, A.M., Deuchars, J., 1994. Temporal and spatial properties of local circuits in neocortex. Trends Neurosci. 17, 119.

Thomson, A.M., Deuchars, J., 1997. Synaptic interactions in neocortical local circuits: dual intracellular recordings in vitro. Cereb. Cortex 7, 510.

Tsodyks, M.V., Markram, H., 1997. The neural code between neocortical pyramidal neurons depends on neurotransmitter release probability. Proc. Natl. Acad. Sci. USA 94 (2), 719.

Wall, M.J., 2005. Short-term synaptic plasticity during development of rat mossy fiber to granule cell synapse. European J. Neurosci. 21, 2149.

Zucker, R.S., Regehr, W.G., 2002. Short-term synaptic plasticity. Trends Neurosci. $64,355$. 